\title{
Consensus-based technical recommendations for clinical translation of renal BOLD MRI
}

\author{
Octavia Bane ${ }^{1}$ (1) losif A. Mendichovszky ${ }^{2} \cdot$ Bastien Milani $^{3} \cdot$ llona A. Dekkers $^{4} \cdot$ Jean-Francois Deux ${ }^{5}$. \\ Per Eckerbom ${ }^{6} \cdot$ Nicolas Grenier $^{7} \cdot$ Michael E. Hall ${ }^{8} \cdot$ Tsutomu Inoue $^{9} \cdot$ Christoffer Laustsen $^{10} \cdot$ Lilach O. Lerman $^{11}$. \\ Chunlei Liu ${ }^{12}$. Glen Morrell ${ }^{13}$. Michael Pedersen ${ }^{14} \cdot$ Menno Pruijm $^{15}$. Elizabeth A. Sadowski ${ }^{16}$. Erdmann Seeliger ${ }^{17}$. \\ Kanishka Sharma ${ }^{18} \cdot$ Harriet Thoeny ${ }^{19} \cdot$ Peter Vermathen ${ }^{20} \cdot$ Zhen J. Wang ${ }^{21} \cdot$ Zbigniew Serafin $^{22}$. Jeff L. Zhang ${ }^{23}$. \\ Susan T. Francis ${ }^{24}$ - Steven Sourbron ${ }^{18}$. Andreas Pohlmann ${ }^{25}$. Sean B. Fain ${ }^{26}$. Pottumarthi V. Prasad ${ }^{27}$
}

Received: 5 August 2019 / Revised: 18 October 2019 / Accepted: 22 October 2019 / Published online: 25 November 2019

(c) The Author(s) 2019

\begin{abstract}
Harmonization of acquisition and analysis protocols is an important step in the validation of BOLD MRI as a renal biomarker. This harmonization initiative provides technical recommendations based on a consensus report with the aim to move towards standardized protocols that facilitate clinical translation and comparison of data across sites. We used a recently published systematic review paper, which included a detailed summary of renal BOLD MRI technical parameters and areas of investigation in its supplementary material, as the starting point in developing the survey questionnaires for seeking consensus. Survey data were collected via the Delphi consensus process from 24 researchers on renal BOLD MRI exam preparation, data acquisition, data analysis, and interpretation. Consensus was defined as $\geq 75 \%$ unanimity in response. Among 31 survey questions, 14 achieved consensus resolution, 12 showed clear respondent preference (65-74\% agreement), and 5 showed equal (50/50\%) split in opinion among respondents. Recommendations for subject preparation, data acquisition, processing and reporting are given based on the survey results and review of the literature. These technical recommendations are aimed towards increased inter-site harmonization, a first step towards standardization of renal BOLD MRI protocols across sites. We expect this to be an iterative process updated dynamically based on progress in the field.
\end{abstract}

Keywords BOLD MRI $\cdot$ Kidney $\cdot$ Imaging $\cdot$ Biomarkers $\cdot$ Standardization $\cdot$ Consensus

\section{Introduction}

Blood oxygenation level dependent (BOLD) MRI, as the name suggests, is an MRI contrast mechanism that depends on the oxygenation status of blood, specifically the oxygenation of blood hemoglobin $(\mathrm{Hb})$ [1]. Oxygen saturation of $\mathrm{Hb}$ changes the magnetic properties of the $\mathrm{Hb}$ from being diamagnetic when fully oxygenated to paramagnetic in its deoxygenated state, which shortens the observed transverse free induction decay time constant, $T_{2}{ }^{*}$, or lengthens the corresponding rate constant $R_{2}{ }^{*}=1 / T_{2}{ }^{*}$. Thus, a change in the ratio of oxy- to deoxy-Hb in blood results in a measurable change on BOLD MRI contrast and is widely used in functional MRI of the brain [2]. Since the ratio of oxy- to

Pottumarthi V. Prasad

pprasad@northshore.org

Extended author information available on the last page of the article
deoxy-Hb is a major determinant of blood partial pressure of oxygen $\left(\mathrm{PO}_{2}\right)$, the method in principle can be used to measure an index of blood $\mathrm{PO}_{2}$. If one assumes that blood $\mathrm{PO}_{2}$ is in a dynamic equilibrium with the surrounding tissue $\mathrm{PO}_{2}$, then the BOLD MRI measurements can reflect tissue $\mathrm{PO}_{2}$. To reflect these underlying assumptions about the biodistribution of $\mathrm{PO}_{2}$, the BOLD MRI $T_{2}{ }^{*}$ or $R_{2} *$ measure is sometimes referred to as "oxygen availability". The method is completely non-invasive, since it utilizes an endogenous contrast mechanism and does not require injection of any exogenous contrast agent.

In contrast to virtually all other organs in the body, in the healthy kidney, not only the supply of oxygen, but also oxygen consumption is determined by blood flow [3]. The major determinant of oxygen consumption in the kidneys is sodium reabsorption. The amount of sodium to be reabsorbed is proportional to the glomerular filtration rate (GFR) which in turn, in general, changes in parallel with renal blood flow. 
Since both oxygen supply and demand are related to renal blood flow, evaluating renal oxygen status independent of perfusion is necessary in the kidneys. Much of the early data on renal oxygenation was obtained in animal models using invasive probes which have documented a gradient of decreasing oxygenation from cortex to inner medulla [4]. However, due to the lack of a non-invasive method for use in humans, very little was known about whether human kidneys have the same anatomical variations in oxygenation as observed in rodents. With the availability of BOLD MRI the presence of such a gradient was verified in the kidneys of healthy subjects [5]. Much of the early literature on BOLD MRI focused on the medulla, a region of naturally low oxygen tension. Since this region is known to be at risk for ischemic injury, understanding the endogenous mechanisms that maintain oxygenation status and effects of exogenous maneuvers that can modulate the oxygenation status were foci of early studies [6-10].

An early application of the BOLD MRI method to clinical studies was in renal artery stenosis where it was important to know who may benefit from re-vascularization [11]. Based on earlier reports in rodent models of diabetes [12,13], there have also been studies assessing the relative oxygen status in individuals with diabetes and varying levels of renal function $[14,15]$. With gaining interest in the chronic hypoxia hypothesis related to progressive chronic kidney disease (CKD) [16], there has been growing interest in evaluating renal oxygenation status in CKD [17-19] and kidney transplant [20-23]. This evolution towards clinical translation has led to the realization of the importance of standardization or harmonization of subject preparation, data acquisition and analysis methods. Without harmonization, it is currently difficult to objectively compare BOLD $T_{2}{ }^{*}\left(R_{2}{ }^{*}\right)$ measures between sites and this constitutes a barrier for multi-center studies and pooling of clinically relevant data to inform future practice.

All physiological and functional imaging methods are inherently dependent on the physiological status of the organ at the time of data acquisition. To be able to compare data from one individual to another it is important to standardize the baseline physiological status (to the best extent possible). Water intake or hydration status has been shown to significantly influence renal oxygenation [6] as does salt intake [9]. Since consumption of food inherently involves consumption of water, fasting prior to the BOLD MRI study (e.g. $4 \mathrm{~h}$ ) is commonly used. Controlling salt intake requires more regimented protocols and it is not practical to implement for routine clinical use.

While early studies used single-shot echo planar imaging (EPI) sequences for renal BOLD MRI [5], the most widely used current method is the multiple gradient echo (mGRE) (Fig. 1a) acquisition [24, 25]. Typically $8-16$ gradient echo images (Fig. 1) are acquired during breath-holds and a

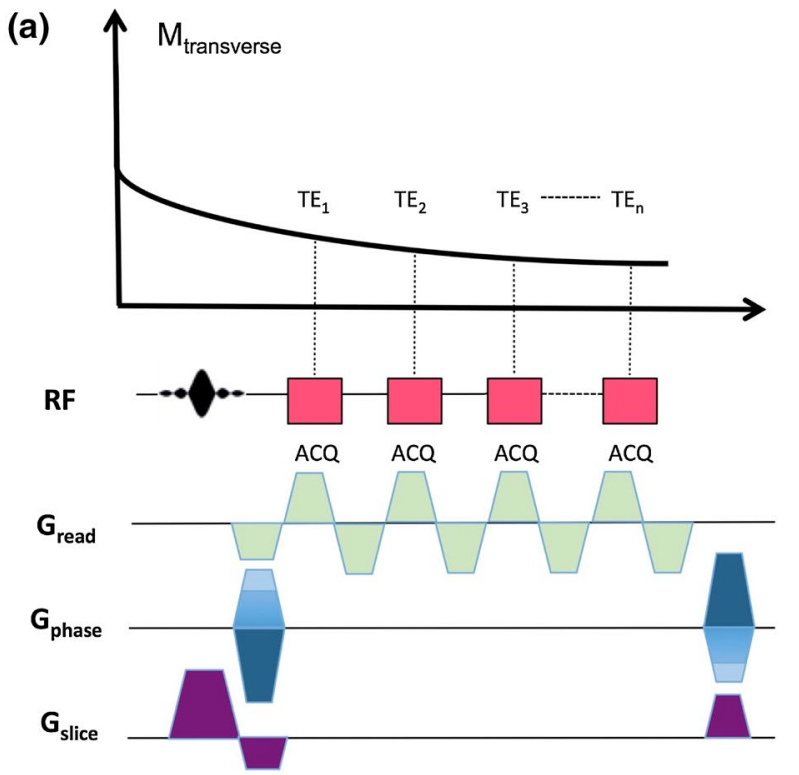

(b)

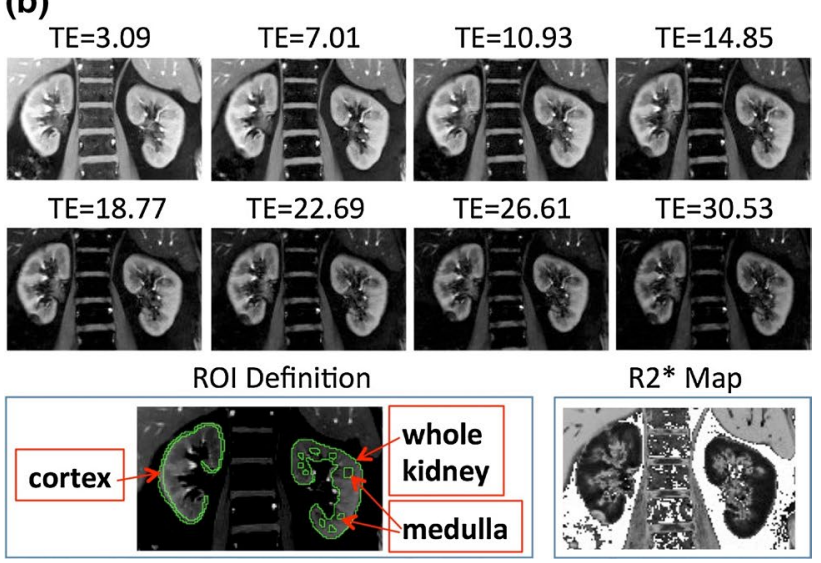

Fig. 1 a Pulse sequence diagram of a multiple gradient-echo (mGRE) sequence with $n$ echo times $\left(\mathrm{TE}_{1}-\mathrm{TE}_{n}\right)$ obtained from a single RF excitation, with the readout gradient $\left(G_{\text {read }}\right)$ rewinded after each signal sampling (ACQ). b BOLD-MRI acquisition (breath hold, 2D mGRE sequence, $\mathrm{TR}=51 \mathrm{~ms}$, flip angle $=30^{\circ}, 5 \mathrm{~mm}$ slice thickness, $5 \mathrm{~mm}$ space between slices, FOV $400 \times 400 \mathrm{~mm}$, matrix $256 \times 256$ ) example with eight echo times (TE from 3.09 to $30.53 \mathrm{~ms}$, bandwidth $256 \mathrm{~Hz} /$ pixel); cortex and medulla ROI delineation example, and $R_{2}{ }^{*}$ grayscale map on a $3 T$ Siemens Skyra ${ }^{\text {fit }}$ in a healthy volunteer (female, age 64, BMI 25.6). (Courtesy: Lu-Ping Li, Ph.D., NorthShore University Health System, Evanston, IL, USA). BOLD bloodoxygen level dependent, FOV field of view, $m G R E$ multi-echo gradient echo, $R F$ radio frequency, $R O I$ region of interest, $T E$ echo time

mono-exponential fit of the signal intensity with respect to echo time is used to estimate the relaxation time constant $T_{2}{ }^{*}$. The sequence is widely available on all major vendor platforms and it typically comes with the option to calculate $T_{2}^{*}$ or $R_{2}^{*}$ online. While this has facilitated the widespread utility of the method, variations in the specific acquisition parameters make the comparison of data across sites difficult. Similarly, the subjective definition of manually defined 
regions of interest (ROIs) is widely reported and leads to an additional source of bias and variability, especially when related to the renal medulla (Fig. 1b). This problem of subjectivity is further aggravated in diseased kidneys since loss of cortico-medullary contrast is a hallmark in several forms of renal disease [26], including CKD. This has led to proposals to perform whole kidney analysis where the user only needs to manually trace the outer boundaries of renal parenchyma [19, 27, 28].

The objective for this initiative is to provide technical recommendations for renal BOLD MRI based on a consensus between experts in the field, aiming to promote a more standardized approach that will facilitate comparison of data across sites. We used a recently published statement paper [29], which included a detailed summary of parameters in their supplementary material, as the starting point to identify topics of interest in developing the questionnaires for seeking consensus. The work was facilitated by the 'PARENCHIMA' initiative "MRI Biomarkers for CKD" (CA16103), a community-driven Action of the European Cooperation in Science and Technology (COST) program of the European Union, which aims to improve the reproducibility and standardization of renal MRI biomarkers.

\section{Materials and methods}

\section{Description of the survey process}

During the PARENCHIMA Annual Plenary Meeting in Prague (October 4-5th 2018), the Task Force on Technical Recommendations for Clinical Renal MRI decided to follow a modified Delphi method [30-32] for seeking consensus regarding technical BOLD MRI acquisition and analysis. The Delphi method derives consensus recommendations through qualitative evaluation of published studies by experts. Where published data is scarce, experts can make inferences based on data from similar contexts, or their own experiences. Published evidence is summarized in the form of a questionnaire. Participants are invited to respond to the questionnaire in several rounds (typically 3 ), reviewing an anonymous summary of the previous responses before responding to the subsequent rounds. Respondents are asked to indicate whether they consider themselves sufficiently knowledgeable to answer each of the questions. Consensus on a question is usually defined as $68 \%-80 \%$ unanimity in responses [31-33]. Discussion in a face-to-face meeting between all respondents usually follows one or several of the rounds, and serves to build consensus in the areas where prior responses failed to achieve consensus. Owing to wide geographic distribution of the experts and respondents, we have adopted a modified Delphi method for this project with only a single face-to-face meeting before the final round of surveys.

\section{Construction of the questionnaire}

The questionnaires were constructed by the panel co-chairs (PVP, OB and SF), between February 2019 and April 2019, and administered online to participants in two rounds via an online form (Google Forms, https://docs.google.com/forms). In constructing the questionnaires, the co-chairs used a recently published PARENCHIMA statement paper [29] and its detailed summary of parameters in the supplementary material, to formulate questions on renal BOLD MRI research topics, technical image acquisition parameters and analysis methods. Round 1 consisted of 35 questions with multiple-choice responses, with the possibility to abstain or to provide a long form answer. Participants were prompted to explain the reasoning behind their choices in a comment section following each item, and also to suggest questions for inclusion in Round 2 of the questionnaire.

Six members of the panel attended a face-to-face meeting between March 18th-19th 2019 at Aarhus University Hospital, Denmark. Based on a review of the first round of questionnaire responses, they worked together on additional questions and on the wording for specific questions in Round 2. Round 2 consisted of 31 items with answers in simplified multiple-choice format ("I agree", "I disagree", "I have insufficient experience to make a recommendation") and possibility to explain the choice in a comment section following each item. Questions that reached consensus in Round 1 were summarized to the respondents in Round 2, and respondents were offered opportunity to comment if they did not agree. Respondents were encouraged to answer the questionnaire based on published evidence (some items pointed to specific publications) and best practices as reflected in the literature, not necessarily on their current research practice. Both iterations of the questionnaire had items grouped under four themes: (1) patient preparation (common items among all PARENCHIMA task force panels for Round 2, see covering review paper); (2) acquisition; (3) analysis; (4) application and interpretation.

\section{Panel selection}

The first panel consisted of 10 PARENCHIMA task force members with a publication record and research interest in BOLD MRI. To expand our pool of respondents for the 2nd round, we invited other experts outside of our collaboration. We identified renal BOLD experts on the basis of a PUBMED literature search using [BOLD MRI] AND [kidney] AND [human]. An additional 16 respondents were enrolled while two respondents to the first questionnaire did not participate in the second one. For the second and final round of 
the questionnaire, we had 24 respondents from 22 academic institutions, 9 countries and 3 continents. All respondents are listed as authors of this manuscript. Panelists were asked to list their specialty when responding to the questionnaire.

\section{Interpretation of the survey results}

At the Aarhus meeting, a "traffic light" system was adopted to issue recommendations based on the degree of consensus achieved through the questionnaire. Green light (consensus, closed issues) on a topic was defined as at least $75 \%$ unanimity in responses to a question. An "orange light" was defined in the case where the responses showed clear opinions (65-74\%), but did not meet the threshold. For a "red light" topic, the questionnaire showed no clear expert preference $(50-64 \%)$, so that no recommendation is possible with the information given to the respondents. It was also agreed that when calculating percentage of responses, the "abstain" responses (i.e. "I have insufficient experience to make a recommendation") will be excluded. However, the percentage of "abstain" responses for each item will be reported, to reflect the level of familiarity of the experts with the topic and to show the topic's importance (or lack thereof) in the clinical renal BOLD MRI community.

\section{Results}

\section{Results of survey}

The final results of the survey are summarized for each area in Tables 1, 2, 3, 4, 5, 6 and 7. For the second and final round of the survey, physicists (MR physicists or biomedical engineers) and clinicians (radiologists, nephrologists or physiologists) were equally represented (Table 2). The panelists reached consensus on 14 out of 31 items (45\%) (Tables 3, 4, 5, 6). Additionally, panelists concurred on the nine questions for which consensus was achieved in Round 1 (Table 7). 12 items met the "orange light" definition, showing a clear direction of respondents' opinions, while 5 items were "red light" topics with a 50/50\% split in respondents' opinions. Even though the overall response did not reach the 75\% threshold, some questions had responses that reached the threshold in one of the sub-group analysis, Physicists or Clinicians. The presentation of responses by sub-group may
Table 1 Final recommendations on renal BOLD MRI data acquisition, analysis and interpretation

\begin{tabular}{|c|c|}
\hline & BOLD MRI \\
\hline Preparation & Normal hydration $(\mathbf{1 0 0} \mathrm{ml}$ water $), 4 \mathrm{~h}$ fasting from food \\
\hline Field strength & 1.5 $\mathrm{T}$ or $3.0 \mathrm{~T}, 3 T$ preferred if available \\
\hline Sequence & 2D mGRE \\
\hline Orientation & Coronal oblique to kidneys \\
\hline In-plane resolution & $2-3 \mathrm{~mm}$ \\
\hline Slice thickness & $3-5 \mathrm{~mm}$ \\
\hline Coverage & $3-5$ slices centered on renal hilum \\
\hline Parallel imaging factor & 2 \\
\hline Fat suppression & Yes \\
\hline $\mathrm{TR}(\mathrm{s})$ & $60-75 \mathrm{~ms}$ \\
\hline $\mathrm{TE}(\mathrm{ms})$ & $\begin{array}{l}8-16 \text { echoes, up to } 50 \mathrm{~ms}\left(\sim T_{2}{ }^{*} \text { cortex }\right) \text { at } 3 T \text { with } \\
\text { choice of in phase for fat-water }\end{array}$ \\
\hline Averages & 1 \\
\hline Breathing mode & Breath hold \\
\hline Image quality control & Recommended \\
\hline ROI placement & Manual \\
\hline Cortical ROI & 1 stripe/slice; $>3$ slices \\
\hline Medullary ROI & 3 samples/slice; $>3$ slices \\
\hline Fitting & Monoexponential or log-linear \\
\hline Reporting & Cortex and medulla \\
\hline Reported metric & $\boldsymbol{R}_{\mathbf{2}}^{*}\left(\mathrm{~s}^{-\mathbf{1}}\right)$ \\
\hline Metric statistics reporting & Mean, median, standard deviation, ROI size \\
\hline Map format & Color or grayscale quantitative map \\
\hline
\end{tabular}

Entries in bold font are based on the consensus process

Bold blood-oxygen level dependent, $R O I$ region of interest, $T_{2} *$ transverse free-induction decay time, $T E$ echo time 
Table 2 Panel characteristics

\begin{tabular}{lll}
\hline Respondents for Round 1 & $n=10$ & Percentage \\
\hline Physicists & $\mathbf{7}$ & $\mathbf{7 0}$ \\
Clinicians & $\mathbf{3}$ & $\mathbf{3 0}$ \\
Radiologists & 1 & 10 \\
Nephrologists & 2 & 20 \\
Physiologists $^{\mathrm{a}}$ & 1 & 10 \\
\hline Respondents for Round 2 & $n=24$ & Percentage \\
\hline Physicists $_{\text {Clinicians/physiologists }}$ & $\mathbf{1 2 ( 5 0 \% )}$ & $\mathbf{5 0}$ \\
Radiologists & $\mathbf{1 3 ( 5 0 \% )}$ & $\mathbf{5 4}$ \\
Nephrologists & 11 & 46 \\
Physiologists & 2 & 8 \\
\hline
\end{tabular}

The co-chairs sought equal representation of MR physicist and clinician researchers involved in BOLD MRI

${ }^{\mathrm{a}} 1$ radiologist was also a $\mathrm{Ph} . \mathrm{D}$. physicist; 1 nephrologist was also a $\mathrm{PhD}$ physiologist

be useful for our readers to draw perspectives, and so we have included sub-group results in all our tables.

\section{Final recommendations}

Our final recommendations, summarized in Table 1, are based on the literature, the consensus views and opinion trends that emerged from the survey and the face-to-face meeting.

- Regarding patient preparation, irrespective of the level of consensus reached, users should consider ways of normalizing the baseline physiological status. Even though data exists only for hydration status, food intake commonly is associated with fluid intake and a likely confounding factor. When feasible, fasting for $4 \mathrm{~h}$ may be a good option. A related issue of bowel gas has not yet been fully resolved in terms of minimizing its presence.

- Even though the choice of $3 T$ did not reach the $75 \%$ threshold, there is a general acceptance that, if given a choice between the field strengths, $3 T$ is preferable. In the absence of access to $3 T, 1.5 T$ remains an adequate choice. While there is a concern for increased magnetic field inhomogeneities at $3 T$, there is considerable experience to-date that supports the higher sensitivity and signal-to-noise ratio (SNR) afforded by $3 T$, making it a preferred choice.

- Magnetic field inhomogeneities (e.g. due to poor shimming, presence of metal or air interfaces) play an important role and should be minimized. These will affect the measured $R_{2}{ }^{*}$ values and results in a voxel-size dependence of $R_{2}{ }^{*}$. Slice thickness is the largest contributor to voxel size, and has the largest contribution to the voxelsize dependence of $R_{2}{ }^{*}$ due to through-slice dephasing although the effects may be lower in the coronal orientation. While smaller and isotropic voxels are preferred, they are limited by the need for breath-holding. Adopting a fixed voxel size for all studies may be a preferred way to standardize the protocol and allow comparisons across sites. This is challenging to implement in routine practice because voxel size can be set directly on the scanners of one vendor (e.g. Philips), while in other vendors' scanners (e.g. Siemens, GE) the voxel size is derived from field of view (FOV) and matrix size. Even if matrix size is kept constant, MRI technologists are inclined to change FOV based on the body habitus. Thus, standard operating procedures (SOPs) are necessary and the MRI technologist needs to be instructed not to change the FOV when prescribing renal BOLD MRI.

- The presence of fat may have an effect on measured $R_{2}{ }^{*}$ values, and use of fat saturation or water-excitation pulses is preferred.

- Most studies to-date have focused on the renal medulla which has a higher $R_{2}^{*}\left(\sim 30 \mathrm{~s}^{-1}\right.$ at $\left.3 T\right)$ and hence a shorter $T_{2}^{*}(\sim 33 \mathrm{~ms})$. In comparison, the renal cortex has $R_{2}{ }^{*} \sim 20 \mathrm{~s}^{-1}$ at $3 T$ and $T_{2}{ }^{*} \sim 50 \mathrm{~ms}$. After fixing the longest TE $=50 \mathrm{~ms}$, investigators need to decide the number of echo times. For a robust acquisition, any number of echoes between 8 and 16 would be acceptable. The actual number of echoes that is realizable will depend on readout bandwidth, echo spacing and image resolution. The bandwidth should be kept to around $300 \mathrm{~Hz} /$ pixel. With this echo time, use of either a mono-exponential or log-linear fit would be acceptable since the noise floor will not be reached.

- $R_{2}{ }^{*}$ was the preferred metric and there was consensus for evaluating both cortical and medullary regions. ROI analysis was preferred, only because of widespread avail- 


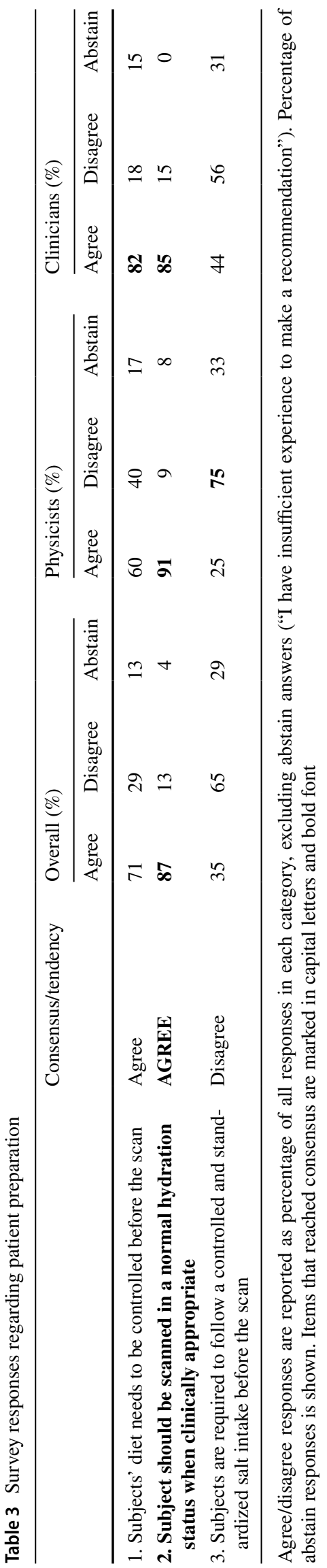

ability. While newer custom methods have advantages such as better objectivity, these also have several limitations. Until automated segmentation becomes routinely available, manual segmentation may be preferred.

- There was an agreement that BOLD MRI provides a qualitative measure of relative oxygen availability and may be most suited for evaluating acute changes within the same kidney, e.g. following administration of furosemide. However, caution was advised when comparing different cohorts because of potential confounding effects such as blood volume fraction, oxy-Hb dissociation curve, and haematocrit [34]. Even though there was consensus in terms of reproducibility at a single site, similar data for multi-site studies remains lacking, and is thus desirable for future studies.

- Similar recommendations are made for BOLD in native kidneys and allografts. However, the allografts' positioning in the iliac fossa, closer to the skin during an endexpiration breath-hold, sometimes requires different mitigation strategies for the tissue-air interface susceptibility artefacts (e.g. placing a bag of saline on the skin over the expected region of the allograft before placing the imaging coil on the subject). Planning of slices in true coronal (to the allograft) orientation is somewhat more difficult than with native kidneys and in some studies an oblique sagittal slice with respect to the allograft' longitudinal axis is preferred.

\section{Discussion}

\section{Patient preparation}

Three identical items on patient preparation prior to the MRI scan (Table 3) were included in all PARENCHIMA panels' questionnaires on clinical renal MRI. To-date, these questions are most relevant for the BOLD MRI panel. A previous systematic review conducted by PARENCHIMA [29] showed that of 20 studies on drug and dietary effects on renal BOLD MRI, 12 studies required their subjects to abstain from food and water for $12 \mathrm{~h}$ before MRI, with ( 8 studies) and without (4 studies) subsequent water loading, 1 study had a shorter fasting period of $2 \mathrm{~h}$ before MRI, and 7 studies did not require fasting. The variety of patient preparation in published studies was reflected in the responses to our survey. The respondents did not reach consensus on the need to control subjects' diet before the scan, although $82 \%$ of clinicians were in favor of controlling the diet. Respondents' comments to the question may explain the lack of consensus:

- $25 \%$ of respondents disagreed that diet should be controlled, 


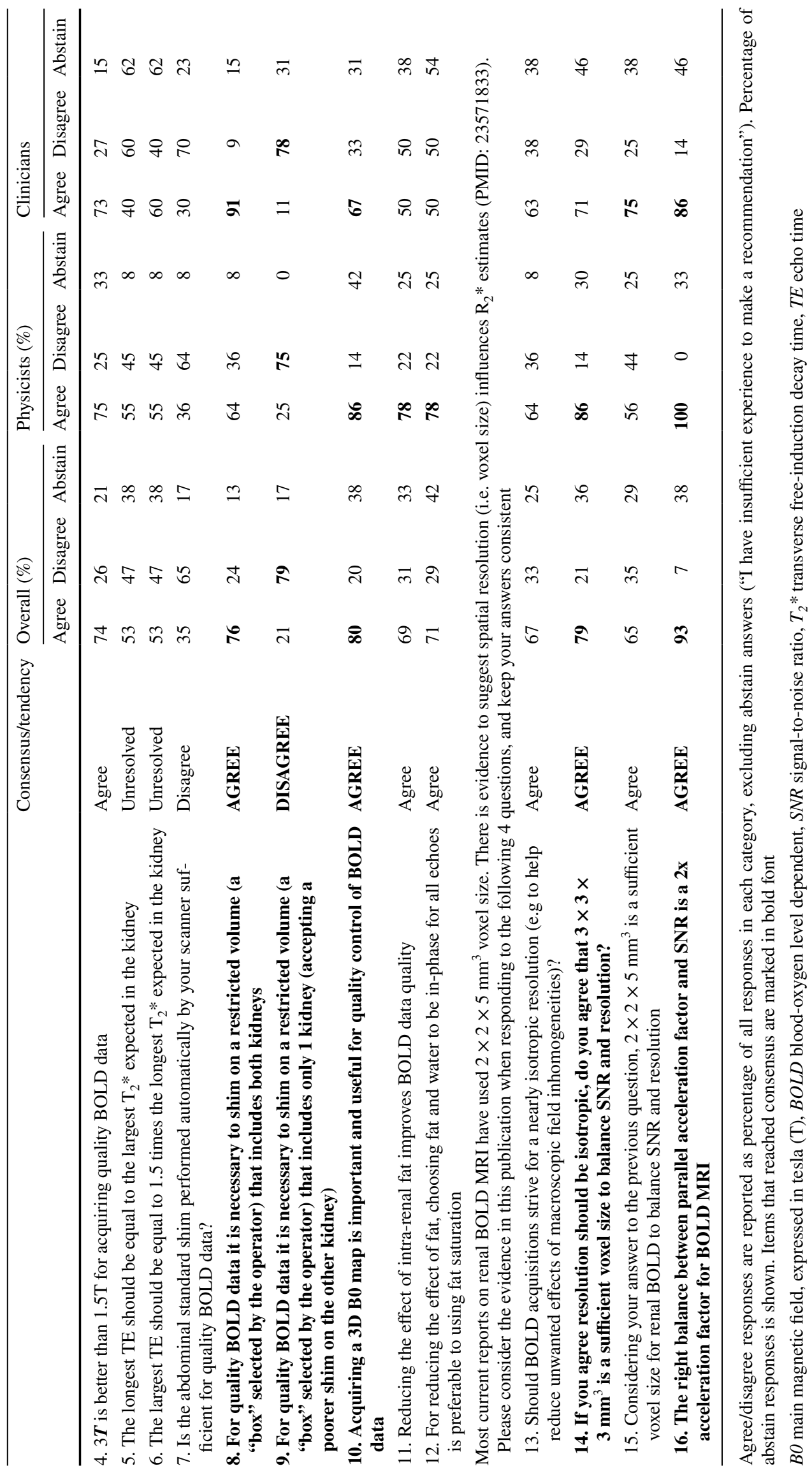




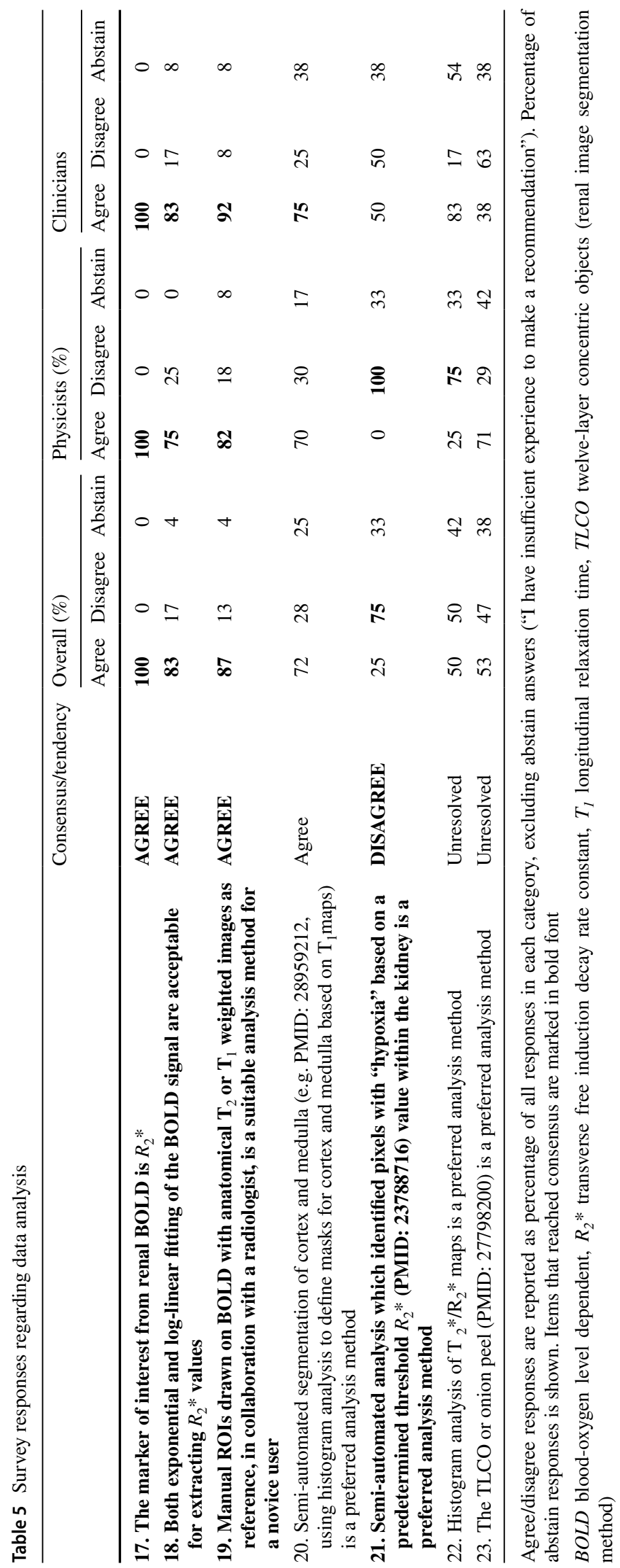




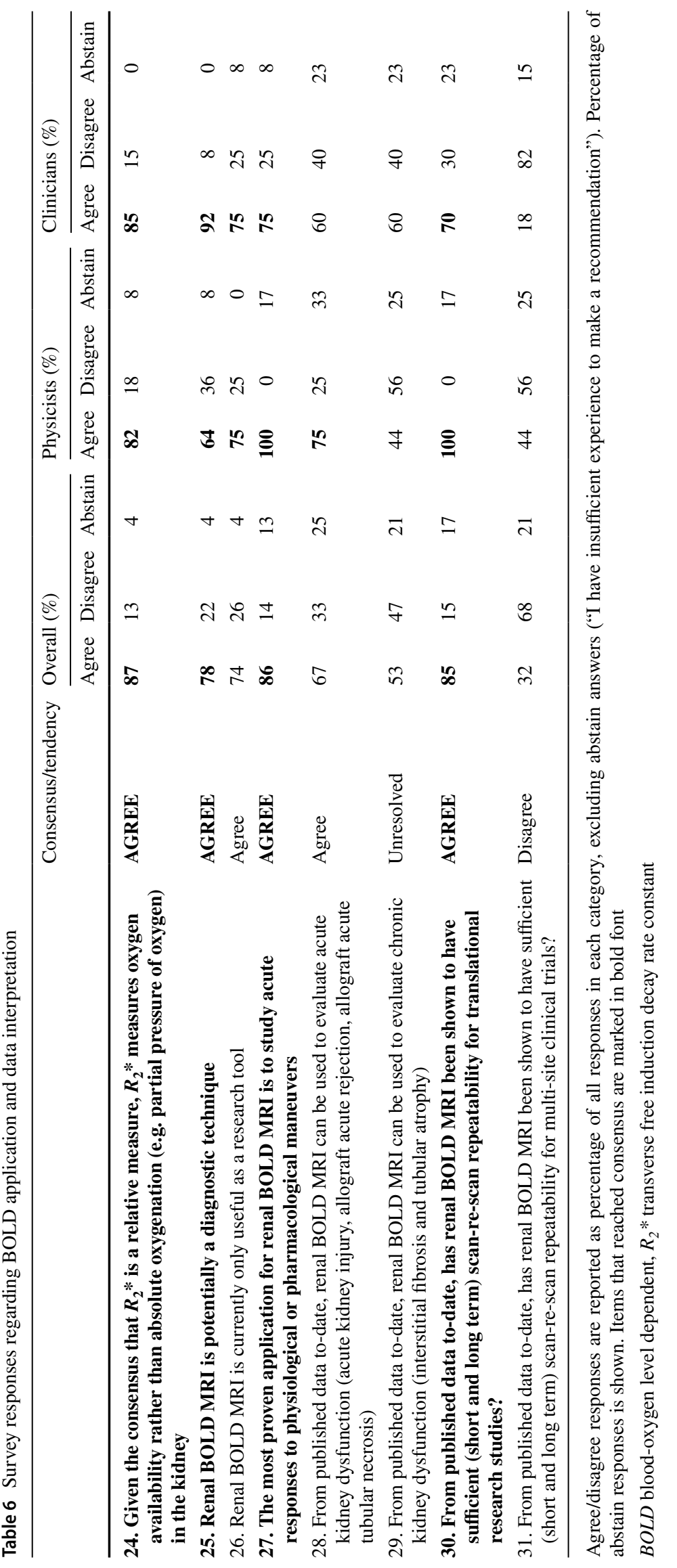


Table 7 Survey items from Round 1 which achieved consensus, excluding abstain ("Do not know") and "Other" answers

$1.5 T$ is acceptable for obtaining quality BOLD data. [choose a single option]

Agree (comment in other)

Disagree (comment in other)

Do not know

Other

The smallest bandwidth should be chosen for the selected maximum TE

Agree (comment in other)

Disagree (comment in other)

Do not know

Other

Do number of echoes and/or spacing between TE's have an effect on data quality?

Agree (comment in other)

Disagree (comment in other)

Do not know

Other

Is breath-holding the best approach for controlling motion in renal BOLD MRI?

Yes

No

Do not know

Other

Is analysis of cortex and medulla important?

Yes, both equally important

Cortex is more important than medulla (comment in other)

Medulla is more important than cortex (comment in other)

Do not know

Other

Is renal BOLD MRI potentially a prognostic technique?

Yes

No

Do not know

Other

Does renal BOLD MRI reflect intra-renal oxygenation (qualitatively or quantitatively)?

Yes, BOLD MRI can quantify intrarenal oxygenation

Yes, qualitatively, there are too many confounding factors for it to be quantitative

No, it does not

Do not know

Other

From existing data, do you believe renal BOLD MRI is more suited to detect changes in renal oxygenation within the same kidney rather than comparing cohorts?

Yes, best used to detect changes within the same kidney, due to confounding effects of blood volume and hematocrit that are different between patients

It is suitable to compare cohorts without adjustments for patients' blood volume and hematocrit

It is suitable to compare cohorts with adjustment for patients' blood volume and hematocrit

Do not know

Other

Does the furosemide stress test/ physiological challenge have value?

Yes, always

Yes, but it may not be necessary or suitable for some applications (comment in Other)

No (comment in Other)

Do not know

Other

These items and the dominant response percentages were shown to participants in Round 2, who concurred to the consensus in Round 1 Bold blood-oxygen level dependent, $T E$ echo time 
- $4 \%$ cited lack of publications showing an effect of diet on BOLD signal, and

- $4 \%$ thought that monitoring and reporting subjects' diet is sufficient.

- $12.5 \%$ of respondents recommended fasting (water only intake) for 4-6 h before the scan, $8 \%$ went further and recommended abstaining from high protein foods $24 \mathrm{~h}$ before the scan in addition to fasting, to minimize susceptibility artifacts from bowel gas.

- Another $8 \%$ of respondents thought restricting drinking before the scan can reduce artifacts from the bowel, and can have an effect on BOLD quality.

- Another $8 \%$ of respondents recommended controlling sodium intake, possibly through standardized meals for study subjects in prospective studies.

The respondents reached consensus (87\%) that patients should be scanned in a normal (i.e. not restricted to water intake) hydration status, based on studies that show the influence of hydration status on BOLD signal (as mentioned by $8 \%$ of respondents). Respondents agreed that hydration status should be controlled, through fasting (4\%) or standardized (i.e. all subjects treated similarly in terms of hydration [35]) intake of water (8\%).

There was no consensus that sodium intake should be standardized before the scan. $65 \%$ of respondents tended to disagree. Even though disagreement among physicists reached consensus threshold (75\%), there was a split opinion among clinicians (agree/disagree: $44 \% / 56 \%$ ). Eight percent of the respondents mentioned their awareness of studies showing that salt intake influences BOLD measurements, with the caveat that the influence of sodium and hydration on the BOLD signal is smaller in the cortex than in the medulla. Modifying salt intake also influences kidney physiology, and may thus confound studies looking at subjects' typical renal physiology, as commented by another $8 \%$ of respondents. $12.5 \%$ of respondents motivated their disagreement to this item by the practical difficulties of controlling subjects' sodium intake, as salt is found in most processed foods. $8 \%$ of respondents recommended that controlled sodium intake is feasible for in-patient subjects who can follow a standardized diet during their hospital stay. While standardizing salt intake is not possible or suitable for the study design, monitoring and recording salt intake (e.g. via food diaries) by the subjects can add to any one study's rigor.

\section{Data acquisition}

The results of the survey items on data acquisition are summarized in Tables 4 and 7. We sought expert opinion on aspects of BOLD MRI data acquisition protocol including choice of field strength, reduction of B0 inhomogeneities, reducing the effect of intra-renal fat, choice of spatial resolution, magnitude and number of echo times (TE) and control of respiratory motion.

Renal BOLD MRI in humans has been performed at both 1.5 and $3 T$ in almost equal distribution to-date: of 79 renal BOLD MRI human and animal studies included in a previous PARENCHIMA systematic review, 41 were performed at 1.5T, 42 at $3 T$, and four used magnets of both field strengths [29]. Even though there was consensus (Table 7; $100 \%$ of non-abstaining respondents, with $30 \%$ abstention rate) that $1.5 T$ provides BOLD data of acceptable quality, there was a general preference for $3 T$ over $1.5 T$ (Table 4 ) (74\% agreement). $3 T$ has the known advantage of greater SNR, increased $T_{2}{ }^{*}$ weighting and hence higher $R_{2}{ }^{*}$ values. However, magnetic field inhomogeneities, bulk magnetic susceptibility (BMS) artifacts and fat-water chemical shift are also magnified at $3 T$ [29]. Twenty percent of respondents mentioned these disadvantages of $3 T$ in imaging the kidney near complex abdominal structures like the bowel, when motivating their disagreement or hesitancy to agree with the statement that $3 T$ was superior. For control of respiratory motion, consensus was reached already in Round 1 (Table 7) that breath-holding is the best approach for both native kidneys and transplants (80\%), although panelists who disagreed (20\%) were familiar with or working on respiratory-triggered sequences.

There was consensus in Round 1 in choosing the smallest receiver bandwidth for the selected maximum TE (and number of echoes) in order to maximize SNR, and most respondents (88\%) thought that the number of echoes and spacing between TEs have an effect on data quality (Table 7). It was generally agreed that more acquired TEs can improve robustness of $T_{2} * / R_{2}{ }^{*}$ fitting. Eight to sixteen echoes are commonly used in the literature to-date. Expert opinion was evenly split regarding choice of longest TE based on the longest $T_{2}{ }^{*}$ expected in the kidney (Table 4 ). While $53 \%$ of non-abstaining respondents tended to agree that the longest TE should be equal to the longest $T_{2}{ }^{*}$ there were concerns that it may be too conservative (e.g. more data can be acquired beyond that value to improve the fit). Conversely, acquiring up to 1.5 times the longest $T_{2}{ }^{*}$ expected in renal tissue, while preferable to $53 \%$ of respondents, raised concerns that the noise floor could be reached in tissues with low oxygenation, that the TR, and hence breath-hold interval, would be increased too much, or that good image quality could not be obtained with some coils at $\mathrm{TE}=1.5$ times longest $T_{2}{ }^{*}$ expected in the kidney.

With regards to mitigating (or minimizing) $\mathrm{B}_{0}$ inhomogeneities, most respondents (65\%, Table 4) did not consider the standard abdominal shim performed by the scanner to be sufficient for quality BOLD data. There was overall consensus that a restricted shim should be performed on a volume encompassing both kidneys ( $76 \%$ of all respondents, $91 \%$ of clinicians, but only $64 \%$ of physicists, agreed), but not 
on a smaller volume encompassing each kidney separately (79\% of all respondents, and $75 \%$ of physicists and $78 \%$ of clinicians, disagreed). The main concern with shimming on each kidney separately was the time consuming workflow (12.5\% of respondents), strongly localized shimming introducing artifacts from outside the shimmed area (8\% of respondents), while 1 respondent observed no difference in $T_{2}{ }^{*}$ maps between the kidneys when shimming for each kidney separately. Respondents using BOLD in the study of kidney transplants used saline bags placed over the skin at the location of the allograft to mitigate susceptibility artifacts from the allograft's proximity to the body surface and from bowel gas $[21,23]$. Respondents acknowledged that localized shimming could not always address $\mathrm{B}_{0}$ inhomogeneities, which is why there was consensus that acquiring a $3 \mathrm{D}$ $\mathrm{B}_{0}$ map is useful for quality control of BOLD data $(80 \%$ of non-abstaining respondents, $78 \%$ of physicists, and $67 \%$ of clinicians agreed). Acquisition of many BOLD datasets with $\mathrm{B}_{0}$ maps should also foster the development of widely available open-source post-processing software tools that correct the measured $R_{2} *$ by removing the effect of $\mathrm{B}_{0}$ inhomogeneity facilitating multicentre studies. Among physicians $67 \%$ of non-abstaining respondents agreed on the question of the choice of $\mathrm{B}_{0}$ map, and there was a high rate of abstaining answers (31-42\%), which suggests a low availability or utilization of $\mathrm{B}_{0}$ mapping sequences and techniques on clinical scanners. However, a dual-echo GRE scheme to generate $\mathrm{B}_{0}$ maps from the phase difference of the two echoes is now available on all MR vendors. It should be noted that there are no reports in the published literature yet to support these recommendations regarding shimming, which may explain the high rate of abstain responses.

One manifestation of $\mathrm{B}_{0}$ inhomogeneity is the voxel-size dependence of $R_{2} *$ estimates [36]. There was evidence to show that slice thickness (usually larger than in-plane voxel size) contributed more to the observed effect [36]. The panelists were asked to consider this evidence [36] when responding to the last four items of the Acquisition portion of the survey (Table 4). While consensus was not achieved on the need for a nearly isotropic resolution, a sizeable portion of non-abstaining respondents (67\%) thought isotropic voxels were preferred for imaging an organ with complex structures like the kidney. However, isotropic imaging raised concerns because of the need to match voxels in multi-parametric protocols with other MRI contrasts that are not best suited to an isotropic resolution protocol, such as arterial spin labeling (ASL). There was consensus for using $3 \times$ $3 \mathrm{~mm}^{2}$ in-plane resolution (not necessarily isotropic). Similarly consensus was reached for using acceleration factor of 2 to achieve anatomical coverage within a breath-hold while maintaining sufficient SNR.

Studies in the liver demonstrate that the presence of fat can influence $T_{2}{ }^{*} / R_{2} *$ measurements [37]. While healthy kidneys do not contain intra-renal fat, in some diseased states such as diabetes [38] and obesity [39], there may be intra-renal fat present. For this and other reasons (delineation of kidney), fat saturation/water excitation is desirable for BOLD MRI acquisitions, but not widely used. The low utilization of these techniques is reflected in the response to the question of whether it is preferable to control for the presence of fat by selection of all echo times with fat-water in phase rather than by performing fat saturation ( $71 \%$ agreement, $42 \%$ abstain rate). The statement reached agreement among physicists (78\%), but not among clinicians (50/50\% split, with $38-54 \%$ abstain rate). Because of the lack of consensus, but tendency towards a choice of in-phase fat-water TEs, we recommend performing both fat saturation/suppression and choosing in-phase TEs.

\section{Data analysis}

The results of the survey items on data analysis are summarized in Tables 5 and 7. 100\% of respondents agreed that the reported value should be $R_{2}{ }^{*}$ (rather than $T_{2}{ }^{*}$ ) obtained from exponential or log-linear fit of BOLD signal (83\%). However, respondents cautioned that the results are not comparable between fitting methods, and that the log-linear fit increases the influence of noise for later echoes with low SNR. A possible solution to correcting for noise at later echoes is to use a weighted log-linear fit. An exponential fit should also be corrected for noise, after identifying the noise floor. Of course, this concern can be mitigated by limiting the maximum TE to be $\sim T_{2}{ }^{*}$ of tissue of interest.

In Round 1, respondents reached consensus that measuring $R_{2}{ }^{*}$ values in both the cortex and medulla is equally important (Table 7; 90\%). Early renal BOLD MRI research was driven by the low oxygenation and greater sensitivity to changes in oxygen demand in the medulla. Some studies recommended the medulla-to-cortex ratio in $R_{2}{ }^{*}$ to be a more sensitive marker especially in diabetes, taking advantage of the disparate changes with disease severity in cortex vs. medulla [14]. Increased $R_{2}{ }^{*}$ in the cortex has been observed in chronic kidney disease (CKD) [19], with either minimal change or actually a reduction in medullary $R_{2}{ }^{*}$ $[15,40,41]$. The consensus among all respondents (Table 5; $82 \%$ ) was that a novice BOLD researcher can obtain $R_{2}$ * measurements in both cortex and medulla using regions of interest (ROIs) drawn in collaboration with a radiologist. $R_{2}{ }^{*}$ measurements in this case can be based on average signal in the ROI (for protocol optimization), or pixel-based within the ROIs. Ideally, manual ROIs should delineate as much of the cortex and medulla as possible, avoiding lesions, large vessels and fat.

More than with image acquisition, there was greater difficulty in obtaining consensus on advanced, semi-automatic image analysis methods. This is due to the lack of availability 
and experience with each method beyond the proponent site. Among advanced methods of ROI segmentation, semi-automated segmentation of cortex and medulla using histogram analysis to define masks for cortex and medulla based on $T_{1}$ maps [42] nearly reached consensus among $72 \%$ of respondents (75\% of clinicians, $70 \%$ of physicists). Histogram analysis of $T_{2}{ }^{*} / R_{2}{ }^{*}$ maps and the 12-layer concentric objects (TLCO) or "onion peel" method [19] generated a 50/50 split in preferences among respondents. There was agreement among physicists (75\%), but not among clinicians (50-50\% split of opinion) that semi-automated analysis methods, which identify pixels as being "hypoxic" based on a predetermined threshold value for $R_{2} *$ [28], are not preferred. Concerns regarding implementation, as it is difficult (if not impossible, given the effects of shimming) to decide on a reliable threshold for hypoxia for the condition or patient population studied. Some common concerns expressed by respondents regarding semi-automated segmentation methods were that segmentation software packages are not widely available, have been tested only with particular acquisition protocols (e.g. preference for true coronal to the kidney orientation for the TLCO method [19]), may not work so well in diseased kidneys with poor cortico-medullary differentiation, and have not been validated across sites.

\section{Application and interpretation}

The results of the survey items on BOLD applications and data interpretation are summarized in Tables 6 and 7. In Round 1 (Table 7), consensus (80\%) was achieved among respondents that renal BOLD MRI can reflect oxygenation qualitatively, as the confounding factors of perfusion, blood volume fraction (per tissue volume), hematocrit, and shifts of the oxy-Hb dissociation curve preclude it from being truly quantitative. For that reason, respondents consented to the statements that renal BOLD MRI is more suited to detect changes in renal oxygenation within the same kidney rather than for comparing cohorts (88\%), and that examining changes in $R_{2}{ }^{*}$ brought on by a furosemide challenge is useful, although not necessary for all applications (75\%).

The consensus statement emerging from Round 1 that BOLD MRI provides a relative measure of oxygen availability, rather than an absolute measure of oxygenation, was supported in Round 2 (Table 6; 87\% of respondents). The consensus from Round 1 that BOLD MRI is a potentially prognostic technique (100\% non-abstaining respondents) was further improved in Round 2, with $78 \%$ of respondents (Table 6) considering BOLD a potentially diagnostic technique. There was notable split among clinicians (92\%) and physicists $(63 \%)$ on the diagnostic value of BOLD. The statement that BOLD MRI is currently only useful as a research tool (Table 6) narrowly missed the consensus threshold with $74 \%$ overall. Respondents commented that BOLD can only be diagnostic in combination with other methods, due to the confounding factors, and that its clinical impact has to be better demonstrated beyond a few experienced centers.

Among applications of BOLD MRI, the consensus (Table 6; 86\% overall) emerged that renal BOLD MRI is best suited for studying acute responses to physiological or pharmacological maneuvers (e.g. water or furosemide challenge). Fewer respondents considered renal BOLD suitable for evaluation of acute dysfunction in native kidneys or allografts $(67 \%$ overall, $75 \%$ of physicists, $60 \%$ of clinicians) or chronic renal dysfunction (53\% overall, $44 \%$ physicists, $60 \%$ clinicians). Although there are studies showing differences between groups in acute dysfunction $[43,44]$, respondents $(8 \%)$ commented there are better ways to assess renal dysfunction, and that more work is needed to show clinical relevance of BOLD MRI (8\%). The division in opinion on the utility of BOLD in CKD is based on the published studies, which found increased $R_{2} *$ (lower oxygenation) [14, 45] or decreased $R_{2} *$ [15] in patients with CKD compared to controls, and did not show a significant correlation of $R_{2}{ }^{*}$ with estimated GFR or CKD stage [35, 40, 46].

With regards to short and long-term scan-rescan repeatability, the consensus among respondents (85\%) was that the literature $[47,48]$ shows high repeatability for translational research, within the same site, if shimming and physiological conditions (diet, hydration) are controlled. However, $68 \%$ of respondents disagreed that multi-center repeatability suitable for clinical trials has been shown in the literature to-date. Respondents commented that standardization of protocols and more accumulation of results from multi-center studies is needed.

\section{Issues reaching consensus}

Most items that did not reach consensus are related to split opinions in the literature, or limited data available to-date. The fact that our respondents did not reach consensus on superiority of imaging at $3 T$, standardizing diet, or salt intake reflects the even split among published studies: studies at $1.5 T$ and $3 T$ are equally represented in the literature, and a minority of published studies have standardized salt intake [29]. Split opinion on the longest TE is also due to a lack of systematic comparison studies exploring how feasible it is to image at long TE's ( $1 \times$ or $1.5 \times T_{2}{ }^{*}$ of cortex) on different platforms, with respondents relying on their individual experience. More than with image acquisition, there was greatest difficulty in obtaining consensus on advanced, semi-automatic image analysis methods. This is due to the lack of availability and experience with each method beyond the proponent site. 


\section{Limitations}

The panel of experts that participated in this consensus formation process was of a relatively small size $(n=24)$. However, it included an equal proportion of clinical and technically oriented scientists from groups that have all developed or applied renal BOLD MRI. Given the small sample size, we did not record other factors that may influence responses, such as respondents' age, years of experience in BOLD MRI, and location by country. In future recommendation surveys and papers, we plan to account for how these factors influence the recommendations. Although consensus emerged for acquisition methods, the greatest variability of responses covered analysis and interpretation of data, particularly semi-automatic segmentation of BOLD MRI cortical and medullary data, since only a limited number of dedicated research groups have experience with the advanced analysis methods.

\section{Remaining challenges for future research}

The unresolved issues emerging from this consensus process point to potential avenues for future research. More systematic studies are needed on whether controlling diet beyond a 4-h fast before the scan through standardized diet and salt intake has an effect on renal BOLD MRI. Technical development, validation and dissemination is needed for $\mathrm{B}_{0}$ mapping and related methods to mitigate $\mathrm{B}_{0}$ inhomogeneities and bulk susceptibility artifacts. Similarly, respiratory motion control by breath-holding is a limiting factor, creating a trade-off between the number of echo times or slices acquired, which can be addressed by further development of fast free-breathing BOLD MRI sequences or respiratory motion correction methods. Respiratory motion control beyond breath-holding is essential for achieving whole kidney coverage in BOLD MRI acquisitions.

On the data analysis side, the survey responses underline the need for dedicated renal BOLD MRI post-processing software to facilitate semi-automated image analysis, with validation and dissemination of the software beyond the most experienced sites. The development and validation of (semi-)automatic segmentations methods for renal BOLD MRI data should be encouraged, especially in patient populations with poor cortico-medullary differentiation, where manual selection of cortical and medullary ROIs can be biased.

There is a future role for artificial neural networks/ artificial intelligence (AI) in solving cortex and medulla segmentation problems on BOLD MRI data acquired in patients with renal disease. To date, there are no published studies on $\mathrm{AI}$ in renal BOLD MRI, but artificial neural networks have been used in the brain for quantitative analysis of BOLD MRI and quantitative susceptibility mapping data [49].
AI methods have been used for automatic segmentation of the cortex and medulla for renal volumetry based on $T_{1}$ weighted imaging, with [50] and without gadolinium contrast enhancement [51-53]. The ROIs obtained by automatic segmentation of $\mathrm{T}_{1}$-weighted images can inform segmentation of the cortex and medulla in a multiparametric MRI protocol. We plan to include a literature review as the field develops, and survey questions on $\mathrm{AI}$ in renal BOLD MRI in future iterations of the recommendations. Use of AI requires large training sets of manually annotated, high-quality renal BOLD MRI data, with robust clinical validation and correlation with patient-centric outcomes [54]. Increased quality and standardization in data acquisition is thus needed for the large-scale deployment of AI in renal BOLD MRI. Multicenter studies with harmonized scanning protocols, uniform semi-automatic data analysis, and the development of a phantom with reference cortical and medullary $R_{2}{ }^{*}$ values are high priority for the qualification of BOLD MRI $R_{2} *$ as a renal biomarker. Additional challenges are also likely to emerge with better understanding of the kidney's complex physiology and pathophysiology, such as the degree to which renal functional markers are influenced by the huge range of drugs used to treat renal and systemic diseases, as well as how these measured renal parameters change in multi-morbidity states.

\section{Conclusion}

This collaborative effort was undertaken with the aim of putting forward technical recommendations for facilitating the more widespread use of renal BOLD MRI protocols for clinical research and hopefully their eventual translation to the clinical setting. Based on the current literature and using a modified Delphi method to seek consensus, we have developed a set of recommendations for renal BOLD MRI data collection. The recommendations are to be considered as guidelines to motivate translation and multi-center studies. Given the dynamic nature of physiological imaging methods, techniques are bound to evolve, especially in terms of data acquisition and analysis of renal BOLD MRI. Therefore, we expect and encourage periodic updates of these recommendations.

Acknowledgements The article is based upon work from COST Action Magnetic Resonance Imaging Biomarkers for Chronic Kidney Disease (PARENCHIMA, COST Action CA 16103, www.renalmri.org), supported by COST (European Cooperation in Science and Technology), www.cost.eu. O.B. received funding (2016-2018) from NIH NIDDK individual fellowship 1F32DK109591. K.S. and S.S. received funding from the Innovative Medicines Initiative 2 Joint Undertaking under grant agreement No 115974. S.T.F, I.M and S.S receive funding from the Medical Research Council [grant number MR/R02264X/1]. A.P. and E.S. receive funding from the German Research Council (DFG; Collaborative Research Centre SFB 1365 Renoprotection). M.P. 
receives funding from the Swiss National Science Foundation (FN 320030-169191). H.C.T: receives funding from Swiss National Science Foundation NIZCOZO_177140/1. P.V.P. receives funding from NIDDK R01 DK093793 and R01 DK106557.

Author contributions Study conception and design: OB, PVP, SBF, AP, IAM, BM, SS. Acquisition of data: OB, PVP, SBF. Analysis and interpretation of data: All authors. Drafting of manuscript: OB, PVP, SBF, AP, IAM. Critical revision: All authors.

\section{Compliance with ethical standards}

Conflict of interest All authors declared that they have no conflict of interest.

Open Access This article is distributed under the terms of the Creative Commons Attribution 4.0 International License (http://creativecommons.org/licenses/by/4.0/), which permits unrestricted use, distribution, and reproduction in any medium, provided you give appropriate credit to the original author(s) and the source, provide a link to the Creative Commons license, and indicate if changes were made.

\section{References}

1. Thulborn KR, Waterton JC, Matthews PM, Radda GK (1982) Oxygenation dependence of the transverse relaxation time of water protons in whole blood at high field. Biochim Biophys Acta 714(2):265-270

2. Thulborn KR (2012) My starting point: the discovery of an NMR method for measuring blood oxygenation using the transverse relaxation time of blood water. Neuroimage 62(2):589-593

3. Epstein FH, Agmon Y, Brezis M (1994) Physiology of renal hypoxia. Ann N Y Acad Sci 718:72-81 (discussion 81-72)

4. Brezis M, Agmon Y, Epstein FH (1994) Determinants of intrarenal oxygenation. I. Effects of diuretics. Am J Physiol 267(6 Pt 2):F1059-F1062

5. Prasad PV, Edelman RR, Epstein FH (1996) Noninvasive evaluation of intrarenal oxygenation with BOLD MRI. Circulation 94(12):3271-3275

6. Prasad PV, Epstein FH (1999) Changes in renal medullary pO2 during water diuresis as evaluated by blood oxygenation leveldependent magnetic resonance imaging: effects of aging and cyclooxygenase inhibition. Kidney Int 55(1):294-298

7. Epstein FH, Prasad P (2000) Effects of furosemide on medullary oxygenation in younger and older subjects. Kidney Int 57(5):2080-2083

8. Simon-Zoula SC, Hofmann L, Giger A, Vogt B, Vock P, Frey FJ, Boesch C (2006) Non-invasive monitoring of renal oxygenation using BOLD-MRI: a reproducibility study. NMR Biomed 19(1):84-89

9. Pruijm M, Hofmann L, Maillard M, Tremblay S, Glatz N, Wuerzner G, Burnier M, Vogt B (2010) Effect of sodium loading/depletion on renal oxygenation in young normotensive and hypertensive men. Hypertension 55(5):1116-1122

10. Manotham K, Ongvilawan B, Urusopone P, Chetsurakarn S, Tanamai J, Limkuansuwan P, Tungsanga K, Eiam-Ong S (2012) Angiotensin II receptor blocker partially ameliorated intrarenal hypoxia in chronic kidney disease patients: a pre-/post-study. Intern Med J 42(4):e33-e37

11. Textor SC, Misra S, Oderich GS (2013) Percutaneous revascularization for ischemic nephropathy: the past, present, and future. Kidney Int 83(1):28-40
12. dos Santos EA, Li LP, Ji L, Prasad PV (2007) Early changes with diabetes in renal medullary hemodynamics as evaluated by fiberoptic probes and BOLD magnetic resonance imaging. Invest Radiol 42(3):157-162

13. Ries M, Basseau F, Tyndal B, Jones R, Deminiere C, Catargi B, Combe C, Moonen CW, Grenier N (2003) Renal diffusion and BOLD MRI in experimental diabetic nephropathy. Blood oxygen level-dependent. J Magn Reson Imaging 17(1):104-113

14. Yin WJ, Liu F, Li XM, Yang L, Zhao S, Huang ZX, Huang YQ, Liu RB (2012) Noninvasive evaluation of renal oxygenation in diabetic nephropathy by BOLD-MRI. Eur J Radiol 81(7):1426-1431

15. Wang ZJ, Kumar R, Banerjee S, Hsu CY (2011) Blood oxygen level-dependent (BOLD) MRI of diabetic nephropathy: preliminary experience. J Magn Reson Imaging 33(3):655-660

16. Fine LG, Orphanides C, Norman JT (1998) Progressive renal disease: the chronic hypoxia hypothesis. Kidney Int Suppl 65:S74-S78

17. Inoue $\mathrm{T}$, Kozawa $\mathrm{E}$, Okada $\mathrm{H}$, Inukai K, Watanabe $\mathrm{S}$, Kikuta $\mathrm{T}$, Watanabe Y, Takenaka T, Katayama S, Tanaka J, Suzuki H (2011) Noninvasive evaluation of kidney hypoxia and fibrosis using magnetic resonance imaging. J Am Soc Nephrol 22(8):1429-1434

18. Prasad PV, Thacker J, Li LP, Haque M, Li W, Koenigs H, Zhou Y, Sprague SM (2015) Multi-parametric evaluation of chronic kidney disease by MRI: a preliminary cross-sectional study. PLoS One 10(10):e0139661

19. Milani B, Ansaloni A, Sousa-Guimaraes S, Vakilzadeh N, Piskunowicz M, Vogt B, Stuber M, Burnier M, Pruijm M (2017) Reduction of cortical oxygenation in chronic kidney disease: evidence obtained with a new analysis method of blood oxygenation leveldependent magnetic resonance imaging. Nephrol Dial Transpl 32(12):2097-2105

20. Thoeny HC, Zumstein D, Simon-Zoula S, Eisenberger U, De Keyzer F, Hofmann L, Vock P, Boesch C, Frey FJ, Vermathen $P$ (2006) Functional evaluation of transplanted kidneys with diffusion-weighted and BOLD MR imaging: initial experience. Radiology 241(3):812-821

21. Sadowski EA, Djamali A, Wentland AL, Muehrer R, Becker BN, Grist TM, Fain SB (2010) Blood oxygen level-dependent and perfusion magnetic resonance imaging: detecting differences in oxygen bioavailability and blood flow in transplanted kidneys. Magn Reson Imaging 28(1):56-64

22. Seif M, Eisenberger U, Binser T, Thoeny HC, Krauer F, Rusch A, Boesch C, Vogt B, Vermathen P (2016) Renal blood oxygenation level-dependent imaging in longitudinal follow-up of donated and remaining kidneys. Radiology 279(3):795-804

23. Niles DJ, Artz NS, Djamali A, Sadowski EA, Grist TM, Fain SB (2016) Longitudinal assessment of renal perfusion and oxygenation in transplant donor-recipient pairs using arterial spin labeling and blood oxygen level-dependent magnetic resonance imaging. Invest Radiol 51(2):113-120

24. Prasad PV, Chen Q, Goldfarb JW, Epstein FH, Edelman RR (1997) Breath-hold $R_{2}{ }^{*}$ mapping with a multiple gradient-recalled echo sequence: application to the evaluation of intrarenal oxygenation. J Magn Reson Imaging 7(6):1163-1165

25. Lebon V, Carlier PG, Brillault-Salvat C, Leroy-Willig A (1998) Simultaneous measurement of perfusion and oxygenation changes using a multiple gradient-echo sequence: application to human muscle study. Magn Reson Imaging 16(7):721-729

26. Lee VS, Kaur M, Bokacheva L, Chen Q, Rusinek H, Thakur R, Moses D, Nazzaro C, Kramer EL (2007) What causes diminished corticomedullary differentiation in renal insufficiency? J Magn Reson Imaging 25(4):790-795

27. Ebrahimi B, Gloviczki M, Woollard JR, Crane JA, Textor SC, Lerman LO (2012) Compartmental analysis of renal BOLD MRI data: introduction and validation. Invest Radiol 47(3):175-182 
28. Saad A, Crane J, Glockner JF, Herrmann SM, Friedman H, Ebrahimi B, Lerman LO, Textor SC (2013) Human renovascular disease: estimating fractional tissue hypoxia to analyze blood oxygen level-dependent MR. Radiology 268(3):770-778

29. Pruijm M, Mendichovszky IA, Liss P, Van der Niepen P, Textor SC, Lerman LO, Krediet CTP, Caroli A, Burnier M, Prasad PV (2018) Renal blood oxygenation level-dependent magnetic resonance imaging to measure renal tissue oxygenation: a statement paper and systematic review. Nephrol Dial Transpl 33(suppl_2):ii22-ii28

30. Hsu C-C, Standford BA (2007) The Delphi technique: making sense of consensus. Pract Assess Res Eval 12(10):1-8

31. Muller BG, van den Bos W, Brausi M, Cornud F, Gontero P, Kirkham A, Pinto PA, Polascik TJ, Rastinehad AR, de Reijke TM, de la Rosette JJ, Ukimura O, Villers A, Walz J, Wijkstra H, Marberger M (2014) Role of multiparametric magnetic resonance imaging (MRI) in focal therapy for prostate cancer: a Delphi consensus project. BJU Int 114(5):698-707

32. Taylor SA, Avni F, Cronin CG, Hoeffel C, Kim SH, Laghi A, Napolitano M, Petit P, Rimola J, Tolan DJ, Torkzad MR, Zappa M, Bhatnagar G, Puylaert CAJ, Stoker J (2017) The first joint ESGAR/ ESPR consensus statement on the technical performance of cross-sectional small bowel and colonic imaging. Eur Radiol 27(6):2570-2582

33. Dalkey N, Helmer O (1963) An experimental application of the DELPHI method to the use of experts. Manage Sci 9(3):458-467

34. Prasad PV (2018) Update on renal blood oxygenation leveldependent MRI to assess intrarenal oxygenation in chronic kidney disease. Kidney Int 93(4):778-780

35. Pruijm M, Hofmann L, Piskunowicz M, Muller ME, Zweiacker C, Bassi I, Vogt B, Stuber M, Burnier M (2014) Determinants of renal tissue oxygenation as measured with BOLD-MRI in chronic kidney disease and hypertension in humans. PLoS One 9(4):e95895

36. Rossi C, Sharma P, Pazahr S, Alkadhi H, Nanz D, Boss A (2013) Blood oxygen level-dependent magnetic resonance imaging of the kidneys: influence of spatial resolution on the apparent $R_{2} *$ transverse relaxation rate of renal tissue. Invest Radiol 48(9):671-677

37. Karlsson M, Ekstedt M, Dahlstrom N, Forsgren MF, Ignatova S, Noren B, Dahlqvist Leinhard O, Kechagias S, Lundberg P (2019) Liver $R_{2}$ * is affected by both iron and fat: a dual biopsy-validated study of chronic liver disease. J Magn Reson Imaging. https://doi. org/10.1002/jmri.26601

38. Morrell GR, Zhang JL, Lee VS (2013) Science to practice: renal hypoxia and fat deposition in diabetic neuropathy-new insights with functional renal MR imaging. Radiology 269(3):625-626

39. Ferguson CM, Eirin A, Michalak GJ, Hedayat AF, Abumoawad AM, Saad A, Zhu X, Textor SC, McCollough CH, Lerman LO (2019) Renal adiposity does not preclude quantitative assessment of renal function using dual-energy multidetector CT in mildly obese human subjects. Acad Radiol. https://doi.org/10.1016/j. acra.2018.12.008

40. Vink EE, de Boer A, Hoogduin HJ, Voskuil M, Leiner T, Bots ML, Joles JA, Blankestijn PJ (2015) Renal BOLD-MRI relates to kidney function and activity of the renin-angiotensin-aldosterone system in hypertensive patients. J Hypertens 33(3):597-603 (discussion 603-594)

41. Zhang R, Wang Y, Chen F, Wang Q, Wang Z, Chen Y, Xiao W (2015) Noninvasive evaluation of renal oxygenation in primary nephrotic syndrome with blood oxygen level dependent magnetic resonance imaging: initial experience. J Int Med Res 43(3):356-363

42. Cox EF, Buchanan CE, Bradley CR, Prestwich B, Mahmoud H, Taal M, Selby NM, Francis ST (2017) Multiparametric renal magnetic resonance imaging: validation, interventions, and alterations in chronic kidney disease. Front Physiol 8:696
43. Djamali A, Sadowski EA, Samaniego-Picota M, Fain SB, Muehrer RJ, Alford SK, Grist TM, Becker BN (2006) Noninvasive assessment of early kidney allograft dysfunction by blood oxygen level-dependent magnetic resonance imaging. Transplantation 82(5):621-628

44. Sadowski EA, Fain SB, Alford SK, Korosec FR, Fine J, Muehrer R, Djamali A, Hofmann RM, Becker BN, Grist TM (2005) Assessment of acute renal transplant rejection with blood oxygen level-dependent MR imaging: initial experience. Radiology 236(3):911-919

45. Xin-Long P, Jing-Xia X, Jian-Yu L, Song W, Xin-Kui T (2012) A preliminary study of blood-oxygen-level-dependent MRI in patients with chronic kidney disease. Magn Reson Imaging 30(3):330-335

46. Michaely HJ, Metzger L, Haneder S, Hansmann J, Schoenberg SO, Attenberger UI (2012) Renal BOLD-MRI does not reflect renal function in chronic kidney disease. Kidney Int 81(7):684-689

47. Li LP, Storey P, Pierchala L, Li W, Polzin J, Prasad P (2004) Evaluation of the reproducibility of intrarenal $R_{2} *$ and DeltaR ${ }_{2}^{*}$ measurements following administration of furosemide and during waterload. J Magn Reson Imaging 19(5):610-616

48. Piskunowicz M, Hofmann L, Zuercher E, Bassi I, Milani B, Stuber M, Narkiewicz K, Vogt B, Burnier M, Pruijm M (2015) A new technique with high reproducibility to estimate renal oxygenation using BOLD-MRI in chronic kidney disease. Magn Reson Imaging 33(3):253-261

49. Hubertus S, Thomas S, Cho J, Zhang S, Wang Y, Schad LR (2019) Using an artificial neural network for fast mapping of the oxygen extraction fraction with combined QSM and quantitative BOLD. Magn Reson Med 82(6):2199-2211

50. Yoruk U, Hargreaves BA, Vasanawala SS (2018) Automatic renal segmentation for MR urography using 3D-GrabCut and random forests. Magn Reson Med 79(3):1696-1707

51. Gloger O, Tonnies K, Laqua R, Volzke H (2015) Fully automated renal tissue volumetry in MR volume data using prior-shape-based segmentation in subject-specific probability maps. IEEE Trans Biomed Eng 62(10):2338-2351

52. Gloger O, Tonnies K, Mensel B, Volzke H (2015) Fully automatized renal parenchyma volumetry using a support vector machine based recognition system for subject-specific probability map generation in native MR volume data. Phys Med Biol 60(22):8675-8693

53. Schnurr A-K, Drees C, Schad LR, Zoellner FG (2019) Comparing sample mining schemes for CNN kidney segmentation in $\mathrm{T} 1 \mathrm{w}$ MRI. In: UK Renal Imaging Network ECP (ed) 3rd International Symposium on Functional Renal Imaging. Nottingham, UK, p 156

54. Liu X, Faes L, Kale AU, Wagner SK, Fu DJ, Bruynseels A, Mahendiran T, Moraes G, Shamdas M, Kern C, Ledsam JR, Schmid MK, Balaskas K, Topol EJ, Bachmann LM, Keane PA, Denniston AK (2019) A comparison of deep learning performance against health-care professionals in detecting diseases from medical imaging: a systematic review and meta-analysis. Lancet Digital Health 1(6):e271-e297

Publisher's Note Springer Nature remains neutral with regard to jurisdictional claims in published maps and institutional affiliations. 


\section{Affiliations}

Octavia Bane ${ }^{1}$ (1) losif A. Mendichovszky ${ }^{2}$ - Bastien Milani ${ }^{3} \cdot$ Ilona A. Dekkers $^{4} \cdot$ Jean-Francois Deux ${ }^{5}$. Per Eckerbom ${ }^{6}$. Nicolas Grenier ${ }^{7}$. Michael E. Hall ${ }^{8}$. Tsutomu Inoue ${ }^{9}$. Christoffer Laustsen ${ }^{10}$ - Lilach O. Lerman ${ }^{11}$. Chunlei Liu ${ }^{12}$. Glen Morrell ${ }^{13}$. Michael Pedersen ${ }^{14} \cdot$ Menno Pruijm $^{15}$. Elizabeth A. Sadowski ${ }^{16}$. Erdmann Seeliger ${ }^{17}$. Kanishka Sharma ${ }^{18}$. Harriet Thoeny ${ }^{19} \cdot$ Peter Vermathen ${ }^{20} \cdot$ Zhen J. Wang $^{21} \cdot$ Zbigniew Serafin $^{22}$. Jeff L. Zhang ${ }^{23}$. Susan T. Francis ${ }^{24}$. Steven Sourbron ${ }^{18}$. Andreas Pohlmann ${ }^{25}$. Sean B. Fain ${ }^{26}$. Pottumarthi V. Prasad ${ }^{27}$

1 BioMedical Engineering and Imaging Institute and Department of Radiology, Icahn School of Medicine at Mount Sinai, New York, NY, USA

2 Department of Radiology, Cambridge University Hospitals NHS Foundation Trust, Cambridge University Hospitals NHS Foundation Trust, Addenbrooke's Hospital, Cambridge, UK

3 Center for BioMedical Imaging, Lausanne University Hospital and University of Lausanne, Lausanne, Switzerland

4 Department of Radiology, Leiden University Medical Center, Leiden, The Netherlands

5 Department of Radiology, Groupe Hospitalier Henri Mondor, Créteil, France

6 Department of Radiology, Institution for Surgical Sciences, Uppsala University, Uppsala, Sweden

7 Department of Radiology, Université de Bordeaux, CHU de Bordeaux, Bordeaux, France

8 Department of Medicine, University of Mississippi Medical Center, Jackson, MS, USA

9 Department of Nephrology, Faculty of Medicine, Saitama Medical University, Saitama, Japan

10 The MR Research Center Department of Clinical Medicine, Aarhus University, Aarhus, Denmark

11 Division of Nephrology and Hypertension, Department of Internal Medicine, Mayo Clinic, Rochester, MN, USA

12 Electrical Engineering and Computer Science, and Helen Wills Neuroscience Institute, University of California Berkeley, Berkeley, CA, USA

13 Department of Radiology and Imaging Sciences, University of Utah, Salt Lake City, UT, USA

14 Department of Clinical Medicine-Comparative Medicine Lab, Aarhus University Hospital, Aarhus, Denmark
15 Nephrology and Hypertension Service, Lausanne University Hospital and University of Lausanne, Lausanne, Switzerland

16 Department of Radiology, University of Wisconsin School of Medicine and Public Health, Madison, WI, USA

17 Institute of Physiology, Charité-University Medicine Berlin, Berlin, Germany

18 Imaging Biomarkers Group, Department of Biomedical Imaging Sciences, University of Leeds, Leeds, UK

19 Department of Radiology, Hôpital Cantonal Fribourgois, University of Fribourg, Fribourg, Switzerland

20 Departments for BioMedical Research and Radiology, Inselspital, Universitaetspital Bern, Bern, Switzerland

21 Department of Radiology and Biomedical Imaging, University of California San Francisco Medical Center, San Francisco, CA, USA

22 Department of Radiology, Nicolaus Copernicus University, Collegium Medicum, Bydgoszcz, Poland

23 Athinoula A. Martinos Center for Biomedical Imaging, Massachusetts General Hospital, Harvard Medical School, Boston, MA, USA

24 Sir Peter Mansfield Centre, University of Notthingham, Notthingham, UK

25 Berlin Ultrahigh Field Facility, Max Delbrueck Center for Molecular Medicine in the Helmholtz Association, Berlin, Germany

26 Departments of Biomedical Engineering, Radiology, and Medical Physics, University of Wisconsin, Madison, WI, USA

27 Department of Radiology, Center for Advanced Imaging, NorthShore University Health System, Evanston, IL, USA 